\title{
Restricted cycle factors and arc-decompositions of digraphs
}

\author{
Jorgen Bang-Jensen and Carl Johan Casselgren
}

\section{Linköping University Post Print}

\section{Tweet}

N.B.: When citing this work, cite the original article.

Original Publication:

Jorgen Bang-Jensen and Carl Johan Casselgren, Restricted cycle factors and arcdecompositions of digraphs, 2015, Discrete Applied Mathematics, (193), 80-93.

http://dx.doi.org/10.1016/j.dam.2015.04.020

Copyright: Elsevier

http://www.elsevier.com/

Postprint available at: Linköping University Electronic Press

http://urn.kb.se/resolve?urn=urn:nbn:se:liu:diva-120854 


\title{
Restricted cycle factors and arc-decompositions of digraphs
}

\author{
Jørgen Bang-Jensen* \\ Department of Mathematics \\ University of Southern Denmark \\ DK-5230 Odense, Denmark
}

\author{
Carl Johan Casselgren ${ }^{\dagger}$ \\ Department of Mathematics \\ Linköping University \\ SE-581 83 Linköping, Sweden
}

\begin{abstract}
We study the complexity of finding 2-factors with various restrictions as well as edge-decompositions in (the underlying graphs of) digraphs. In particular we show that it is $\mathcal{N} \mathcal{P}$-complete to decide whether the underlying undirected graph of a digraph $D$ has a 2 -factor with cycles $C_{1}, C_{2}, \ldots, C_{k}$ such that at least one of the cycles $C_{i}$ is a directed cycle in $D$ (while the others may violate the orientation back in $D$ ). This solves an open problem from [J. Bang-Jensen et al., Vertex-disjoint directed and undirected cycles in general digraphs, JCT B 106 (2014), 1-14]. Our other main result is that it is also $\mathcal{N} \mathcal{P}$-complete to decide whether a 2-edge-coloured bipartite graph has two edge-disjoint perfect matchings such that one of these is monochromatic (while the other does not have to be). We also study the complexity of a number of related problems. In particular we prove that for every even $k \geq 2$, the problem of deciding whether a bipartite digraph of girth $k$ has a $k$-cycle-free cycle factor is $\mathcal{N} \mathcal{P}$-complete. Some of our reductions are based on connections to Latin squares and so-called avoidable arrays.
\end{abstract}

Keywords: Cycle factor, 2-factor, mixed problem, NP-complete, Complexity, cycle factors with no short cycles, Latin square, avoidable arrays, monochromatic matchings

\section{Introduction}

Notation not introduced here follows [1,5]. We distinguish between (non-directed) cycles and directed cycles in digraphs, where the former is a subgraph that corresponds to a cycle in the underlying graph of a digraph. The notions of a (non-directed) path and a directed path are defined in a similar way. A cycle factor in a digraph is a spanning subgraph consisting of directed cycles, and a 2-factor in a digraph (or graph) is a spanning subgraph consisting of cycles. We denote by $U G(D)$ the underlying graph of a directed graph $D$.

\footnotetext{
*E-mail address: jbj@imada.sdu.dk. Parts of this work was done while the author attended the program "Graphs, hypergraphs and computing" at Institute Mittag-Leffler, spring 2014. The research of Bang-Jensen was also supported by the Danish research council under grant number 1323-00178B

${ }^{\dagger}$ E-mail address: carl.johan.casselgren@liu.se. Part of the work done while the author was a postdoc at Mittag-Leffler Institute. Research supported by Mittag-Leffler Institute.
} 
In this paper we consider several variations on the problem of finding cycle factors of digraphs. The problems of deciding if a given graph has a 2-factor and if a given digraph has a cycle factor are fundamental problems in combinatorial optimization, and both these problems are well-known to be solvable in polynomial time, see e.g. $[1,5]$. Here, we are particularly interested in problems concerning the complexity of deciding existence of spanning subgraphs that in a sense lies "inbetween" 2-factors and cycle factors. In particular, we answer the question of the complexity of the following two problems by the first author:

Problem 1.1. [3, Problem 3] 2-factor with at least one directed cycle.

Instance: A digraph $D$.

Question: Does $D$ have a 2-factor $F$ such that at least one cycle in $F$ is a directed cycle, while the rest of the cycles do not have to respect the orientations of $\operatorname{arcs}$ in $D$ ?

\section{Problem 1.2. [7] Disjoint perfect matchings one of which is monochromatic}

Instance: A 2-edge colored bipartite graph $B=(U, V ; E)$.

Question: Does $B$ have two edge-disjoint perfect matchings $M_{1}, M_{2}$ so that every edge of $M_{1}$ has color 1 , while $M_{2}$ may use edges of both colors?

This problem is equivalent to the following problem (see [1, Section 16.7]).

\section{Problem 1.3. Semi-directed 2-factors of bipartite digraphs}

Instance: A bipartite digraph $B=(X, Y ; A)$.

Question: Does $U G(B)$ have a 2-factor which is the union of a perfect matching from $X$ to $Y$ in $B$ (respecting the orientation) and a perfect matching in $U G(B)$ ?

Thus we are asking for a collection of cycles covering all vertices of $B$ such that every second edge (starting from $X$ ) is oriented from $X$ to $Y$ in $D$, whereas the remaining edges do not have to respect the orientation of $D$.

The motivation for studying such "mixed" problems for digraphs, that is, problems concerning structures in a digraph $D$ where only part of the structure has to respect the orientation of the arcs of $D$, is that this way one can obtain new insight into the complexity of various problems which have natural analogues for graphs and digraphs. As an example, in $[2,3]$ the problem of deciding for a digraph $D$ the existence of a directed cycle $C$ in $D$ and a cycle $C^{\prime}$ in $U G(D)$ which are vertex disjoint was studied. It was shown that this problem is polynomially decidable for the class of digraphs with a bounded number of cycle transversals of size 1 (vertices whose removal eliminates all directed cycles) and $\mathcal{N} \mathcal{P}$-complete if we allow arbitrarily many transversal vertices. For (di)graphs one can decide the existence of two disjoint (directed) cycles in polynomial time $[12,13]$.

Note that the variant of Problem 1.1 where we ask if $D$ has a 2-factor $F$ such that at most one cycle in $F$ is not directed is $\mathcal{N} \mathcal{P}$-complete. This can easily be proved as follows: It is $\mathcal{N} \mathcal{P}$-complete to decide if a given graph $G$ is Hamiltonian. Let $D^{\prime}$ be a digraph with a cycle factor, and let $D^{\prime \prime}$ be an acyclic orientation of the given graph $G$. Next, let $D$ be the disjoint union of $D^{\prime}$ and $D^{\prime \prime}$ (or add an arbitrary arc between a vertex of $D^{\prime}$ and a vertex of $D^{\prime \prime}$ if one wants $D$ to be connected). Then $G$ is Hamiltonian if and only if $D$ has a 2 -factor where at most one cycle is not a directed cycle.

We show that Problem 1.1 and 1.2 are both $\mathcal{N} \mathcal{P}$-complete.

Theorem 1.4. 2 -factor with at least one directed cycle is $\mathcal{N} \mathcal{P}$-complete. 
Theorem 1.5. Disjoint perfect matchings one of which is monochromatic is $\mathcal{N} \mathcal{P}$-complete.

In fact, in the latter case, we shall prove that this problem is $\mathcal{N} \mathcal{P}$-complete already for bipartite graphs with maximum degree 3 .

Given an $n \times n$ array $A$ where each cell contains a (possibly empty) subset of $\{1,2, \ldots, n\}$, we say that $A$ is avoidable if there is an $n \times n$ Latin square $L$ such that each cell of $L$ does not contain a symbol that appears in the corresponding cell of $A$. We also say that $L$ avoids $A$. If a cell in $A$ is empty, then we also say that this cell contains entry $\emptyset$. In [6] it was proved that determining whether a given array where each cell contains a subset of $\{1,2\}$ is avoidable is $\mathcal{N} \mathcal{P}$-complete. Using Theorem 1.5 we can prove the following strengthening of that result.

Corollary 1.6. The problem of determining whether an array where each cell contains either the set $\{1\}$, the set $\{1,2\}$ or $\emptyset$ is avoidable is $\mathcal{N} \mathcal{P}$-complete.

Proof. We reduce Problem 1.2 to the problem of avoiding an array where each cell contains symbol 1 , the set $\{1,2\}$ or is empty.

Let $G$ be a balanced bipartite graph on $n+n$ vertices with an edge coloring $f$ using colors 1 and 2. Let $\left\{x_{1}, \ldots, x_{n}\right\}$ and $\left\{y_{1}, \ldots, y_{n}\right\}$ be the parts of $G$. We form an $n \times n$ array $A$ from $G$ as follows:

- If $x_{i} y_{j} \notin E(G)$, then we set $A(i, j)=\{1,2\}$.

- If $x_{i} y_{j} \in E(G)$ and $f\left(x_{i} y_{j}\right)=2$, then we set $A(i, j)=\{1\}$.

- If $x_{i} y_{j} \in E(G)$ and $f\left(x_{i} y_{j}\right)=1$, then set $A(i, j)=\emptyset$.

It is straightforward to verify that there are two disjoint generalized diagonals ${ }^{1} D_{1}$ and $D_{2}$ in $A$, where $D_{1}$ has no cell with symbol 1 and $D_{2}$ has no cell with symbol 2 (and therefore a Latin square avoiding $A$ ) if and only if $G$ has two disjoint perfect matchings one of which does not contain any edge with color 2 .

Next, we consider a variant of a problem studied by Hartvigsen: in [10] he proved that the problem of deciding if a bipartite graph has a 4-cycle-free 2-factor (i.e. a 2-factor with no 4-cycle) can be solved in polynomial time. The problem of determining if a general graph has a 2-factor without 3 -cycles is also solvable in polynomial time [11]. The analogous question of the complexity of existence of 2-factors in general graphs where no cycle has length 4 or less is open to the best of our knowledge, while the problem of determining if a graph $G$ has a 2-factor where all cycles have length at least 6 is $\mathcal{N} \mathcal{P}$-complete if $G$ is bipartite, and thus also in the general case (see e.g. [4, 10]).

For digraphs, the problem of determining whether a general digraph has a cycle factor where every cycle has length at least 3 is $\mathcal{N} \mathcal{P}$-complete, see e.g. [4]. Here we consider the analogous question for bipartite digraphs. We strengthen the result of [4] as follows:

Proposition 1.7. The problem of determining if a given bipartite digraph $D$ has a 2-cycle-free cycle factor is $\mathcal{N} \mathcal{P}$-complete.

Using this result we show how to prove the following:

Theorem 1.8. For each even $k \geq 2$, the problem of determining if a bipartite digraph $D$ with no directed cycles of length at most $k$ has a $(k+2)$-cycle-free cycle factor is $\mathcal{N} \mathcal{P}$-complete.

\footnotetext{
${ }^{1} \mathrm{~A}$ generalized diagonal of an $n \times n$ matrix $A$ is a collection of elements $a_{1, \pi(1)}, \ldots, a_{n, \pi(n)}$ where $\pi$ is a permutation of $[n]$.
} 
In particular, the problem of determining if a given oriented bipartite graph has a cycle factor with no 4-cycle is $\mathcal{N} \mathcal{P}$-complete; so the natural analogue of Hartvigsen's positive result on 4-cyclefree 2-factors in bipartite graphs does not hold in the digraph setting.

Finally, we consider decompositions of digraphs. It is well-known that the problem of determining if the edge set of a given graph has a decomposition into edge-disjoint cycles is solvable in polynomial time, as is also the analogous problem of deciding if the arc set of a given digraph has a decomposition into arc-disjoint directed cycles. Here we shall prove the following:

Theorem 1.9. It is $\mathcal{N} \mathcal{P}$-complete to decide for a given digraph $D=(V, A)$ whether there is a decomposition of $A$ into arc-disjoint cycles $C_{1}, \ldots, C_{k}$ of $D$ with the property that at most one of these cycles is not directed.

Note that the opposite problem, where we ask for a decomposition of $A(D)$ into arc-disjoint cycles $C_{1}, \ldots, C_{k}$ of $D$ such that $C_{1}$ is a directed cycle but all the other cycles do not have to respect the orientation of the arcs, is trivial: As we want a decomposition into arc-disjoint cycles of $D$ it follows that $U G(D)$ must be Eulerian. Hence if $D$ contains any directed cycle $C$, this can play the role of $C_{1}$ above, showing that the answer is no if and only if either $D$ is acyclic or $U G(D)$ is not Eulerian.

The rest of the paper is organized as follows: In section 2 we first prove Proposition 1.7 and using this result, we prove Theorem 1.8 and Theorem 1.4. Section 2 is concluded by the proof of Theorem 1.5. Section 3 contains the proof of Theorem 1.9 and in Section 4 we give an instance of Problem 1.2 which can be solved in polynomial time.

\section{Restricted cycle factors}

For the proof of Proposition 1.7 we will use a result on avoiding arrays. As mentioned above, in [6] it was proved that the following problem is $\mathcal{N} \mathcal{P}$-complete:

\section{Problem 2.1. Avoiding multiple-entry arrays with 2 symbols}

Instance: An $n \times n$ array $A$, such that each cell is either empty or contains a subset of the symbols in $\{1,2\}$.

Question: Is $A$ avoidable?

Proof of Proposition 1.7. We shall reduce Problem 2.1 to the problem of deciding if a given bipartite digraph has a 2-cycle-free cycle factor.

Let $A$ be an $n \times n$ array, where each cell is either empty or contains a subset of $\{1,2\}$. We form an $n \times n$ bipartite digraph $D$ with parts

$$
X=\left\{x_{1}, \ldots, x_{n}\right\} \text { and } Y=\left\{y_{1}, \ldots, y_{n}\right\}
$$

by, for all $i, j$, including the $\operatorname{arc}\left(x_{i}, y_{j}\right)$ in the arc set of $D$ if and only if the symbol 1 does not appear in the set $A(i, j)$ in position $(i, j)$ of the array $A$, and including the arc $\left(y_{j}, x_{i}\right)$ if and only if the symbol 2 does not appear in the set $A(i, j)$. We shall prove that $A$ is avoidable if and only if $D$ has a 2-cycle-free cycle factor.

Suppose that $A$ is avoidable, which means that there is a Latin square $L$ avoiding $A$. Consider the generalized diagonals $B_{1}, B_{2}$ in $L$, containing all cells with entries 1 and 2 , respectively. Since $L$ avoids $A$, any cell in $A$ corresponding to a cell in $B_{1}$ does not contain symbol 1 ; and any cell in 
$A$ corresponding to a cell in $B_{2}$ does not contain the symbol 2 . Hence, if $(i, j) \in B_{1}$, then $\left(x_{i}, y_{j}\right)$ is an $\operatorname{arc}$ of $D$; and if $(i, j) \in B_{2}$, then $\left(y_{j}, x_{i}\right)$ is an $\operatorname{arc}$ of $D$. Thus the set of arcs

$$
\left\{\left(x_{i}, y_{j}\right):(i, j) \in B_{1}\right\} \cup\left\{\left(y_{j}, x_{i}\right):(i, j) \in B_{2}\right\}
$$

induces a cycle factor $F$ in $D$, and since $B_{1}$ and $B_{2}$ are disjoint, $F$ is 2-cycle-free.

Conversely, suppose that $D$ has a 2-cycle-free cycle factor $F$. Let $F_{1}$ be the set of arcs going from $X$ to $Y$ in $F$, and let $F_{2}$ be the arcs going from $Y$ to $X$. Define an array $P$ by including the symbol 1 in the set $P(i, j)$ if and only if $\left(x_{i}, y_{j}\right) \in F_{1}$, and including the symbol 2 in the set $P(i, j)$ if and only if $\left(y_{j}, x_{i}\right) \in F_{2}$. Since $F$ is 2-cycle-free, each cell in $P$ has at most one entry. In fact, $P$ is an $n \times n$ partial Latin square where both symbols 1 and 2 occur exactly $n$ times. Thus we may partition the unfilled cells of $P$ into $n-2$ generalized diagonals $B_{3}, \ldots, B_{n}$ and then assign the set $\{i\}$ to all cells of $B_{i}, i=3, \ldots, n$. So $P$ is completable to a Latin square $L$. Moreover, since $\left(x_{i}, y_{j}\right)$ is an arc of $D$ if and only if the symbol $1 \notin A(i, j)$, and $\left(y_{j}, x_{i}\right)$ is an arc of $D$ if and only if the symbol $2 \notin A(i, j), L$ avoids $A$.

$D$
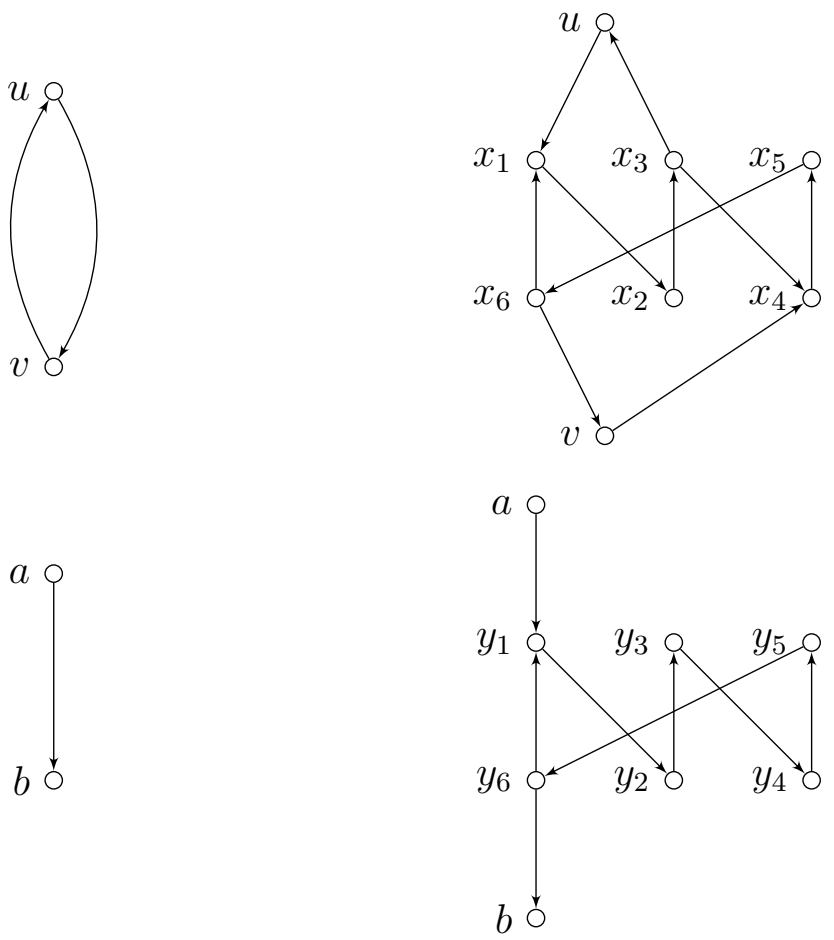

Figure 1: Constructing $D^{\prime}$ from $D$ in the proof of Theorem 1.8.

Proof of Theorem 1.8. We shall prove the theorem in the case when $k=2$, by reducing the problem of existence of 2-cycle-free cycle factors in bipartite digraphs to the problem of existence of 4-cycle-free cycle factors in oriented bipartite graphs. For $k>2$, there is a similar reduction.

Let $D$ be a bipartite digraph. From $D$ we shall form a bipartite digraph $D^{\prime}$ that does not have any 2-cycles as follows: 
(i) For each (directed) 2-cycle $u v u$ of $D$ we do the following: Remove the $\operatorname{arcs}(u, v)$ and $(v, u)$, add 6 new vertices $x_{1}, \ldots, x_{6}$, let $Q=x_{1} x_{2} x_{3} x_{4} x_{5} x_{6} x_{1}$ be a directed 6-cycle joining these vertices, and add $\operatorname{arcs}\left(u, x_{1}\right),\left(x_{3}, u\right),\left(v, x_{4}\right),\left(x_{6}, v\right)$.

(ii) For each arc $(a, b)$ of $D$ which is not in any 2-cycle we do the following: Remove the arc $(a, b)$, add 6 new vertices $y_{1}, \ldots, y_{6}$, let $Q^{\prime}=y_{1} y_{2} y_{3} y_{4} y_{5} y_{6} y_{1}$ be a directed 6 -cycle joining these vertices, and add arcs $\left(a, y_{1}\right),\left(y_{6}, b\right)$.

The graph obtained by repeating the procedure (i) for each 2-cycle of $D$, and the procedure (ii) for each arc of $D$ which is not in any 2-cycle, we denote by $D^{\prime}$ (see Figure 1). It is easily verified that $D^{\prime}$ is bipartite and contains no 2-cycles.

For the construction (i) we say that the $\operatorname{arcs}\left(u, x_{1}\right),\left(x_{3}, u\right),\left(v, x_{4}\right),\left(x_{6}, v\right)$ are the arcs of $D^{\prime}$ corresponding to $(u, v)$ and $(v, u)$. We also say that the 6-cycle $Q$ is the 6-cycle of $D^{\prime}$ associated with uvu.

For the construction (ii) we say that the $\operatorname{arcs}\left(a, y_{1}\right),\left(y_{6}, b\right)$ are the arcs of $D^{\prime}$ corresponding to $(a, b)$. We also say that the directed 6-cycle $Q^{\prime}$ is the directed 6-cycle of $D^{\prime}$ associated with $(a, b)$.

Let us now prove that $D$ has a 2-cycle-free cycle factor if and only if $D^{\prime}$ has a 4 -cycle-free cycle factor. Suppose first that $D$ has a 2-cycle-free cycle factor $F$, and let $C$ be a directed cycle of $F$. Then $C$ defines a directed cycle $C^{\prime}$ in $D^{\prime}$ in the following way:

Let $(a, b)$ be an arc of $C$ that is not in any 2-cycle of $D$, and let $y_{1} y_{2} \ldots y_{6} y_{1}$ be its associated directed 6 -cycle in $D^{\prime}$ along with the arcs $\left(a, y_{1}\right),\left(y_{6}, b\right)$ of $D^{\prime}$ corresponding to $(a, b)$; we define a directed path

$$
P_{1}=a y_{1} y_{2} y_{3} y_{4} y_{5} y_{6} b
$$

in $D^{\prime}$ corresponding to $(a, b)$.

Since $F$ does not contain any directed 2-cycles, we can proceed similarly for any arc in $C$ that is in a 2-cycle: suppose that $(u, v)$ is in the 2-cycle $u v u$ and that $(u, v)$ is in $C$. Assume further that $x_{1} x_{2} \ldots x_{6} x_{1}$ is the associated directed 6-cycle in $D^{\prime}$ and that $\left(u, x_{1}\right),\left(x_{3}, u\right),\left(v, x_{4}\right),\left(x_{6}, v\right)$ are the arcs of $D^{\prime}$ corresponding to $(u, v)$ and $(v, u)$; we define a directed path

$$
P_{2}=u x_{1} x_{2} x_{3} x_{4} x_{5} x_{6} v
$$

in $D^{\prime}$ corresponding to $(u, v)$.

By concatenating all directed paths in $D^{\prime}$ corresponding to $\operatorname{arcs}$ of $C$ in $D$, we obtain a directed cycle $C^{\prime}$ in $D^{\prime}$. Moreover, if $C_{1}$ and $C_{2}$ are disjoint directed cycles in $F$, then the corresponding cycles $C_{1}^{\prime}$ and $C_{2}^{\prime}$ clearly traverses different vertices of $D$ in $D^{\prime}$, so they are disjoint. Let $F^{\prime}$ be a subgraph of $D^{\prime}$ consisting of the collection of cycles in $D^{\prime}$ arising from cycles in $F$ via the above construction. Then $F^{\prime}$ covers all vertices in $V\left(D^{\prime}\right) \cap V(D)$ and each cycle of $F^{\prime}$ clearly has length at least 6. Moreover, the subgraph of $D^{\prime}$ induced by $V\left(D^{\prime}\right) \backslash V(D)$ is a collection of disjoint directed 6-cycles, and if some vertex of such a directed 6-cycle is in $F^{\prime}$, then all vertices of this directed 6-cycle is in $F^{\prime}$. Therefore, $F^{\prime}$ together with the subgraph of $D^{\prime}$ induced by $V\left(D^{\prime}\right) \backslash V\left(F^{\prime}\right)$ forms a cycle factor of $D^{\prime}$. Moreover, each cycle in this cycle factor has length at least 6 .

Suppose now that $D^{\prime}$ has a 4-cycle-free cycle factor $F^{\prime}$. Let $(a, b)$ be an arc of $D$ that is not in any 2-cycle, $y_{1} y_{2} \ldots y_{6} y_{1}$ be the associated directed 6-cycle in $D^{\prime}$, and $\left(a, y_{1}\right)$ and $\left(y_{6}, b\right)$ be the corresponding arcs in $D^{\prime}$. We need the following claim, which easily follows from the facts that all vertices in $\left\{y_{1}, y_{2}, \ldots, y_{5}\right\}$ have out-degree 1 and all vertices in $\left\{y_{2}, y_{3}, \ldots, y_{6}\right\}$ have in-degree 1 .

Claim 2.1. It holds that $\left(a, y_{1}\right) \in A\left(F^{\prime}\right)$ if and only if $\left(y_{6}, b\right) \in A\left(F^{\prime}\right)$. 
We define $E_{1}$ to be the set of all $\operatorname{arcs}(a, b)$ in $D$ that are not in any 2-cycles and satisfying that there are vertices $y_{1}, y_{6} \in V\left(D^{\prime}\right) \backslash V(D)$ such that $\left(a, y_{1}\right) \in A\left(F^{\prime}\right)$ and $\left(y_{6}, b\right) \in A\left(F^{\prime}\right)$. Let $E_{1}^{\prime}$ be the set of all corresponding $\operatorname{arcs}\left(a, y_{1}\right)$ and $\left(y_{6}, b\right)$ in $D^{\prime}$ that are in $F^{\prime}$. It follows from Claim 2.1 that $E_{1}$ induces a subgraph with maximum in- and out-degree at most 1 in $D$, and that a vertex in $D$ has the same in- and out-degree in $D\left[E_{1}\right]$ as in $D^{\prime}\left[E_{1}^{\prime}\right]$.

Next, let $u v u$ be a directed 2-cycle in $D, x_{1} x_{2} \ldots x_{6} x_{1}$ be the associated 6-cycle in $D^{\prime}$, and $\left(u, x_{1}\right),\left(x_{3}, u\right),\left(x_{6}, v\right),\left(v, x_{4}\right)$ be the corresponding arcs in $D^{\prime}$, as in Figure 1 . We will use the following claim which follows easily from the fact that $F^{\prime}$ is a 4-cycle-free cycle factor.

Claim 2.2. (I) If $\left(u, x_{1}\right) \in A\left(F^{\prime}\right)$, then $\left(x_{6}, v\right) \in A\left(F^{\prime}\right)$, and $\left\{\left(x_{3}, u\right),\left(v, x_{4}\right)\right\} \cap A\left(F^{\prime}\right)=\emptyset$.

(II) If $\left(x_{6}, v\right) \in A\left(F^{\prime}\right)$, then $\left(u, x_{1}\right) \in A\left(F^{\prime}\right)$ and $\left\{\left(x_{3}, u\right),\left(v, x_{4}\right)\right\} \cap A\left(F^{\prime}\right)=\emptyset$.

(III) If $\left(x_{3}, u\right) \in A\left(F^{\prime}\right)$, then $\left(v, x_{4}\right) \in A\left(F^{\prime}\right)$ and $\left\{\left(x_{6}, v\right),\left(u, x_{1}\right)\right\} \cap A\left(F^{\prime}\right)=\emptyset$.

(IV) If $\left(v, x_{4}\right) \in A\left(F^{\prime}\right)$, then $\left(x_{3}, u\right) \in A\left(F^{\prime}\right)$ and $\left\{\left(x_{6}, v\right),\left(u, x_{1}\right)\right\} \cap A\left(F^{\prime}\right)=\emptyset$.

We define $E_{2}$ to be the set of all arcs $(u, v)$ in $D$ such that $(u, v)$ is in some 2-cycle, and there are vertices $x, x^{\prime} \in V\left(D^{\prime}\right) \backslash V(D)$ satisfying that $(u, x) \in A\left(F^{\prime}\right)$ and $\left(x^{\prime}, v\right) \in A\left(F^{\prime}\right)$. Let $E_{2}^{\prime}$ be the set of all corresponding arcs $(u, x)$ and $\left(x^{\prime}, v\right)$ in $D^{\prime}$ that are in $F^{\prime}$. It follows from Claim 2.2 that $E_{2}$ induces a subgraph with maximum in- and out-degree at most 1 in $D$, and that a vertex in $D$ has the same in- and out-degree in $D\left[E_{2}\right]$ as in $D^{\prime}\left[E_{2}^{\prime}\right]$. Moreover, there is no 2-cycle in $D\left[E_{2}\right]$.

Furthermore, the sets $E_{1}$ and $E_{2}$ are disjoint, and $E_{1}^{\prime} \cup E_{2}^{\prime}$ contains every arc of $A\left(F^{\prime}\right)$ that is incident with a vertex of $D$; so it follows that the subgraph of $D$ induced by $E_{1} \cup E_{2}$ is a cycle factor with no cycle of length 2 .

Next, we turn to the proof of Theorem 1.4. We shall reduce the problem of deciding existence of 2-cycle-free cycle factors in bipartite digraphs (the problem in Theorem 1.7) to the problem 2-factor with at least one directed cycle. Given a bipartite digraph $D$, we shall construct a digraph $D^{\prime}$ from $D$ and then show that $D$ has a 2-cycle-free cycle factor $F$ if and only if $D^{\prime}$ has a 2-factor $F^{\prime}$ such that at least one cycle of $F^{\prime}$ is a directed cycle of $D^{\prime}$.

Proof of Theorem 1.4. Let $D$ be a bipartite digraph. First we construct the auxiliary digraph $H$ from $D$. Suppose that $D$ has $r$ directed 2-cycles and denote them by $T_{i}=u_{i} v_{i} u_{i}, i=1, \ldots, r$.

(i) For each $\operatorname{arc}(a, b)$ of $D$ that is not in any 2-cycle we do the following: remove the arc $(a, b)$, and let $Q_{a b}$ be an orientation of a non-directed 4-cycle with 4 new vertices $c_{1}, c_{2}, c_{3}, c_{4}$ divided into partite sets $\left\{c_{1}, c_{3}\right\}$ and $\left\{c_{2}, c_{4}\right\}$ where we orient all edges towards $c_{2}$ or $c_{4}$, and add the arcs $\left(a, c_{2}\right)$ and $\left(c_{1}, b\right)$ (see Figure 2).

(ii) For each 2-cycle $u_{i} v_{i} u_{i}$ of $D$ we do the following: remove the $\operatorname{arcs}\left(u_{i}, v_{i}\right)$ and $\left(v_{i}, u_{i}\right)$ and add two disjoint directed 6-vertex paths

$$
L_{i}^{(x)}=x_{1}^{(i)} x_{2}^{(i)} x_{3}^{(i)} x_{4}^{(i)} x_{5}^{(i)} x_{6}^{(i)}
$$

and

$$
L_{i}^{(y)}=y_{1}^{(i)} y_{2}^{(i)} y_{3}^{(i)} y_{4}^{(i)} y_{5}^{(i)} y_{6}^{(i)}
$$

on 12 new vertices, along with the additional $\operatorname{arcs}\left(x_{1}^{(i)}, x_{6}^{(i)}\right)$ and $\left(y_{1}^{(i)}, y_{6}^{(i)}\right)$. Moreover, add the $\operatorname{arcs}\left(u_{i}, x_{4}^{(i)}\right),\left(y_{3}^{(i)}, u_{i}\right),\left(x_{5}^{(i)}, v_{i}\right),\left(v_{i}, y_{2}^{(i)}\right)$ (see Figure 2). 

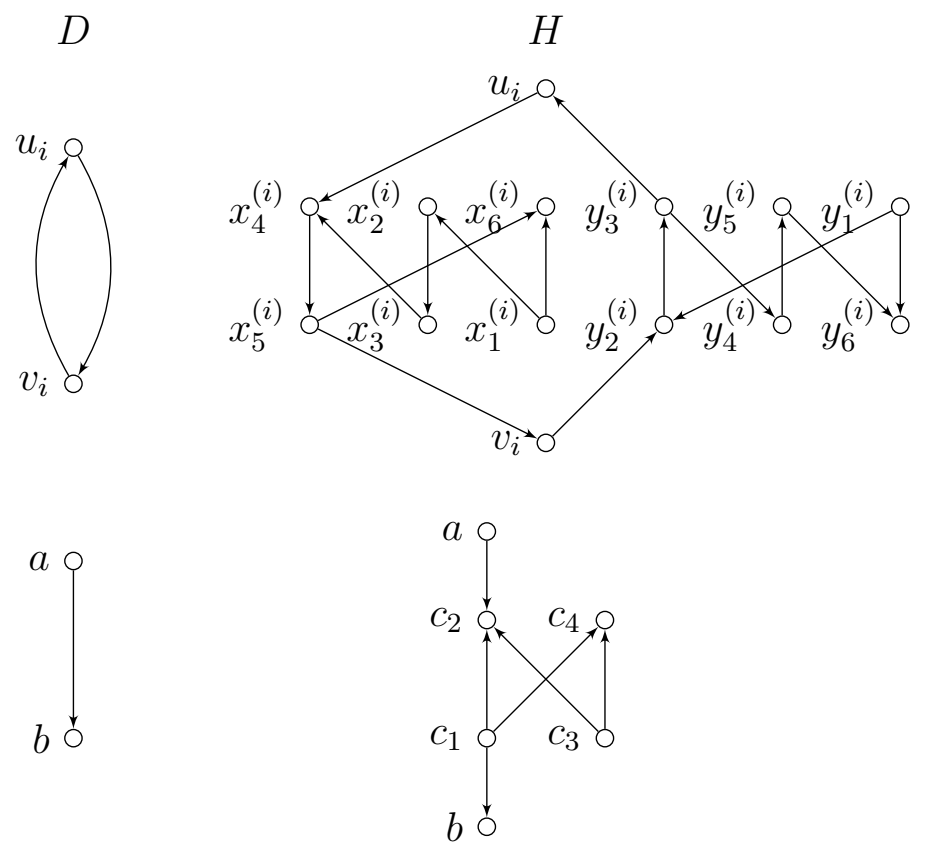

Figure 2: Constructing $H$ from $D$ in the proof of Theorem 1.4. Step 1.

(iii) For each $i=1, \ldots, r$, let $Z_{i}=\left\{z_{1}^{(i)}, z_{2}^{(i)}, z_{3}^{(i)}\right\}$ be a set of three new vertices, and for $i=1, \ldots, r$ (indices taken modulo r) add the arcs in

$$
\left\{\left(z_{1}^{(i)}, z_{2}^{(i)}\right),\left(z_{2}^{(i)}, z_{3}^{(i)}\right),\left(x_{6}^{(i)}, z_{1}^{(i)}\right),\left(y_{6}^{(i)}, z_{1}^{(i)}\right),\left(z_{3}^{(i)}, x_{1}^{(i+1)}\right),\left(z_{3}^{(i)}, y_{1}^{(i+1)}\right)\right\}
$$

(see Figure 3).

Denote the resulting graph by $H$. For the construction (i), we say that $Q_{a b}$ is the 4-cycle associated with $(a, b)$.

Next, we shall construct the graph $D^{\prime}$ from $H$ by proceeding as follows for each vertex $v$ of $V(H) \cap$ $V(D)$ : let $\left\{q_{1}, \ldots, q_{l}\right\}$ be the in-neighbors of $v$ in $H$. For $i=1, \ldots, l$, add a set $\left\{w_{1}^{(i)}, w_{2}^{(i)}, w_{3}^{(i)}\right\}$ of new vertices and replace each arc $\left(q_{i}, v\right)$ with a directed path

$$
q_{i} w_{1}^{(i)} w_{2}^{(i)} w_{3}^{(i)} v
$$

these are called connecting paths between $N_{H}^{-}(v)$ and $v$; moreover, add $l-1$ additional directed 3 -vertex paths

$$
p_{1}^{(1)} p_{2}^{(1)} p_{3}^{(1)}, \ldots, p_{1}^{(l-1)} p_{2}^{(l-1)} p_{3}^{(l-1)}
$$

on altogether $3(l-1)$ new vertices

$$
\left\{p_{1}^{(1)}, p_{2}^{(1)}, p_{3}^{(1)}, \ldots, p_{1}^{(l-1)}, p_{2}^{(l-1)}, p_{3}^{(l-1)}\right\}
$$

these are called non-connecting paths between between $N_{H}^{-}(v)$ and $v$. Next, for each $i=1, \ldots, l-1$ we add the $\operatorname{arcs}\left(p_{1}^{(i)}, w_{3}^{(i)}\right),\left(p_{1}^{(i)}, w_{3}^{(i+1)}\right),\left(w_{1}^{(i+1)}, p_{3}^{(i)}\right),\left(w_{1}^{(i)}, p_{3}^{(i)}\right)$. 


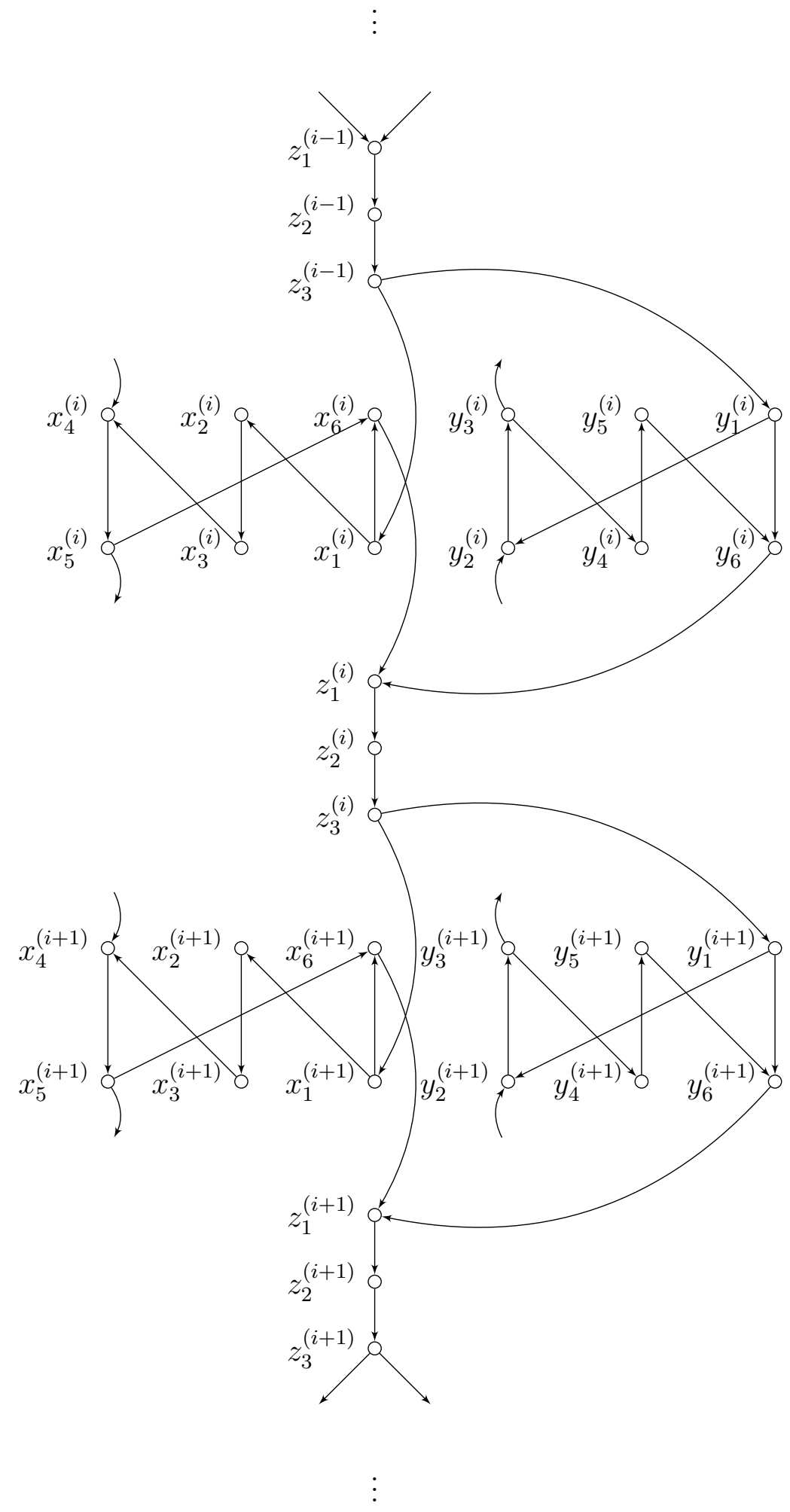

Figure 3: Constructing $H$ from $D$ in the proof of Theorem 1.4. Step 2. 


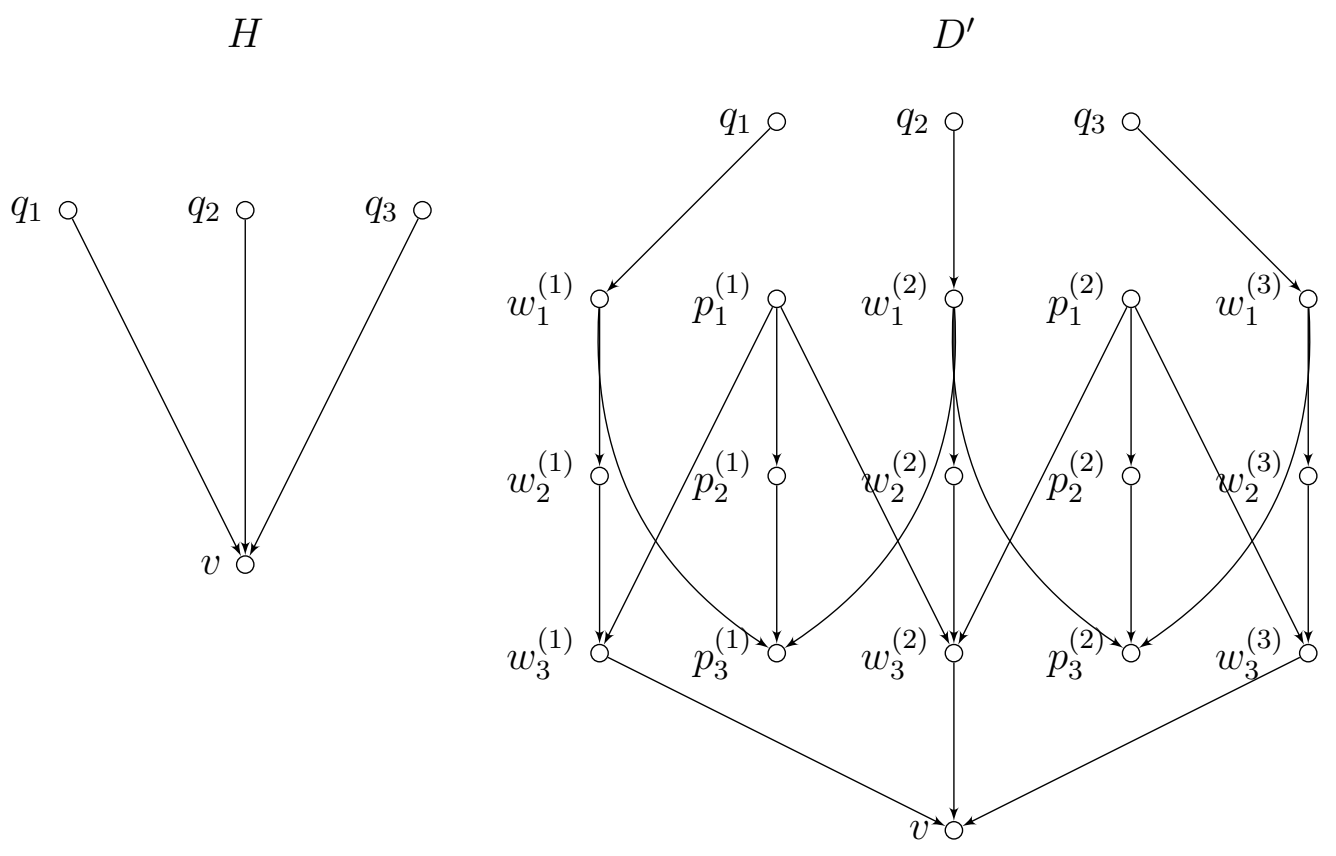

Figure 4: Constructing $D^{\prime}$ from $H$ in the proof of Theorem 1.4.

By repeating this process for every vertex of $V(H) \cap V(D)$ we obtain the digraph $D^{\prime}$ (see Figure 4 for an example). For a vertex $v$ of $V(H) \cap V(D)$, denote by $J_{v}$, the subgraph of $D^{\prime}$ induced by $N_{H}^{-}(v), v$ and all connecting and non-connecting paths between $v$ and $N_{H}^{-}(v)$.

Suppose now that $D$ has a 2-cycle-free cycle factor $F$. We shall form the required 2-factor $F^{\prime}$ in $D^{\prime}$, by first showing how $F$ induces a 2-factor $F_{H}$ in $H$, and then demonstrating how $F_{H}$ yields the required factor $F^{\prime}$ in $D^{\prime}$.

Consider a cycle $C$ in $F$. Then $C$ defines a corresponding cycle $C_{H}$ in $H$ in the following way:

- Suppose that $(a, b)$ is an arc of $D$ that lies on $C$ and is not in any 2 -cycle, and let $\left\{c_{1}, c_{2}, c_{3}, c_{4}\right\}$ be the vertices of the associated 4-cycle of $(a, b)$ as in Figure 2. Then we define a corresponding (non-directed) path $P_{1}=a c_{2} c_{3} c_{4} c_{1} b$ in $H$.

- Suppose that $\left(u_{i}, v_{i}\right)$ is an arc of a 2-cycle that lies on $C$. Then $\left(v_{i}, u_{i}\right) \notin A(C)$, and we define a corresponding (non-directed) path $P_{2}=u_{i} x_{4}^{(i)} x_{3}^{(i)} x_{2}^{(i)} x_{1}^{(i)} x_{6}^{(i)} x_{5}^{(i)} v_{i}$ in $H$. (The case when $\left(v_{i}, u_{i}\right)$ is in $C$ is analogous.)

By concatenating all paths in $H$ corresponding to arcs of $C$ in $D$, we obtain a cycle $C_{H}$ in $H$ covering the same vertices of $V(D)$ as $C$.

It should be clear that the cycles in $F$ in the way described above defines a subgraph $\hat{F}_{H}$ of $H$ that consists of a collection of disjoint cycles, such that none of the cycles in $\hat{F}_{H}$ is a directed cycle in $H$, and each vertex in $D$ has in- and out-degree 1 in $\hat{F}_{H}$. We shall now define a directed cycle $C_{\text {dir }}$ in $H$.

Note that for each $j$, if some vertex of $L_{j}^{(x)}$ is in $\hat{F}_{H}$, then all vertices of $L_{j}^{(x)}$ are in $\hat{F}_{H}$ and no vertex of $L_{j}^{(y)}$ is in $\hat{F}_{H}$. Let $I$ be a maximal subset of indices $i$ of $\{1, \ldots, r\}$ such that no vertex of 
$L_{i}^{(x)}$ is in $\hat{F}_{H}$. Now, we define $\hat{C}_{\text {dir }}$ to be the subgraph of $H$ induced by

$$
\bigcup_{i \in I} V\left(L_{i}^{(x)}\right) \cup \bigcup_{i \in\{1, \ldots, r\} \backslash I} V\left(L_{i}^{(y)}\right) \cup Z_{1} \cup \cdots \cup Z_{r} .
$$

Let us now define

$$
C_{\mathrm{dir}}=\hat{C}_{\mathrm{dir}}-\left\{\left(x_{1}^{(1)}, x_{6}^{(1)}\right), \ldots,\left(x_{1}^{(r)}, x_{6}^{(r)}\right)\right\}-\left\{\left(y_{1}^{(1)}, y_{6}^{(1)}\right), \ldots,\left(y_{1}^{(r)}, y_{6}^{(r)}\right)\right\} .
$$

$C_{\text {dir }}$ is a directed cycle of $H$ disjoint from $\hat{F}_{H}$. Now consider the graph $H^{\prime}=H-V\left(C_{\text {dir }}\right)-V\left(\hat{F}_{H}\right)$. Since each vertex of $D$ has in- and out-degree 1 in $\hat{F}_{H}$ and all vertices of $Z_{1} \cup \cdots \cup Z_{r}$ are in $V\left(C_{\text {dir }}\right)$, $H^{\prime}$ is a collection of disjoint (non-directed) 4- and 6-cycles. Define $F_{H}=\hat{F}_{H} \cup C_{\text {dir }} \cup H^{\prime}$. Then $F_{H}$ is a spanning subgraph of $H$ where each component is a cycle, and exactly one cycle of $F_{H}$ is directed. Moreover, each vertex in $V(D)$ has in- and out-degree 1 in $F_{H}$.

Let us now construct $F^{\prime}$ from $F_{H}$. A cycle $C$ in $\hat{F}_{H}$ translates into a cycle $C^{\prime}$ in $D^{\prime}$ in the following way: If $(s, t)$ is in $C$ and $(s, t) \in A\left(D^{\prime}\right)$, then $t \notin V(D)$, because any in-neighbor in $D^{\prime}$ of a vertex in $V(D)$ is not in $H$ (by the construction of $D^{\prime}$ from $H$ ); and we include $(s, t)$ in $C^{\prime}$; otherwise, if $(s, t)$ is in $C$ and $(s, t) \notin A\left(D^{\prime}\right)$, then $t \in V(D)$ and there is a connecting path $P$ in $J_{t}$ with origin $s$ and terminus $t$. We include the path $P$ in $C^{\prime}$.

It should be clear that the cycles in $\hat{F}_{H}$ in this way defines a subgraph $\hat{F}_{D^{\prime}}$ of $D^{\prime}$ which is a collection of disjoint cycles, and which covers all vertices of $D$. Note further that the cycle $C_{\text {dir }}$ is in $D^{\prime}$ as are also all the cycles in $H^{\prime}$. So it follows from the construction of $D^{\prime}$ from $H$ that $\hat{D}=D^{\prime}-V\left(\hat{F}_{D^{\prime}}\right)-V\left(C_{\text {dir }}\right)-V\left(H^{\prime}\right)$ is a subgraph of $D^{\prime}$ consisting of equally many connecting and non-nonnecting paths between vertices $v \in V(D)$ and their in-neighbors in $H$. It is easy to see that $\hat{D}$ contains a 2 -factor $M$ where every cycle has length 6 , and each cycle contains exactly one nonconnecting path and some vertices of exactly one connecting path. The graph $M \cup C_{\text {dir }} \cup H^{\prime} \cup \hat{F}_{D^{\prime}}$ is the required 2-factor of $D^{\prime}$.

Suppose now conversely that $F^{\prime}$ is a 2-factor of $D^{\prime}$ such that at least one cycle of $F^{\prime}$ is a directed cycle in $D^{\prime}$. Denote by $C_{\text {dir }}$ the directed cycle of $D^{\prime}$ that is in $F^{\prime}$. We prove a series of claims concerning $F^{\prime}$.

Claim 2.3. Let $v \in V(D)$ and consider the subgraph $J_{v}$ of $D^{\prime}$. Let $\left\{q_{1}, \ldots, q_{l}\right\}$ be the in-neighbors of $v$ in $H$. Then exactly one of the arcs in $J_{v}$ that are incident with a vertex in $\left\{q_{1}, \ldots, q_{l}\right\}$ is in $F^{\prime}$; and exactly one of the arcs in $J_{v}$ that are incident with $v$ is in $F^{\prime}$.

The proof of this claim is omitted. It is easily deduced by doing some case analysis using e.g. Figure 4 and the fact that $F^{\prime}$ is a 2-factor.

It follows from Claim 2.3 that $F^{\prime}$ induces a 2-factor $F_{H}$ in $H$ in the following way:

For each arc of $F^{\prime}$ that is in $H$, we include this arc in $F_{H}$; for each vertex $v \in V(D)$, include the $\operatorname{arc}\left(q_{i}, v\right)$ in $F_{H}$, where $q_{i}$ is the unique in-neighbor of $v$ in $H$ that has out-degree 1 in $F^{\prime}$. Obviously, we have that each vertex of $V(D)$ has in- and out-degree 1 in $F_{H}$. Moreover, the directed cycle $C_{\text {dir }}$ clearly corresponds to a directed cycle $C_{\text {dir }}^{(H)}$ in $F_{H}$.

Consider an arc $(a, b)$ in $D$ that is not in any 2-cycle and its associated 4-cycle $Q_{a b}$ in $H$ and label the vertices of $Q_{a b}$ according to Figure 2. It is easy to see that if $\left(a, c_{2}\right)$ is in $F_{H}$, then $\left(c_{3}, c_{2}\right)$ is in $F_{H}$, and thus $\left(a, c_{2}\right)$ is not in $C_{\mathrm{dir}}^{(H)}$.

Now consider an arc $\left(u_{i}, v_{i}\right)$ that is in a 2-cycle of $D$. In $H$, this 2 -cycle is replaced by the directed paths $L_{i}^{(x)}$ and $L_{i}^{(y)}$ and some additional arcs (see Figure 2). It is easy to see that if $\left(u_{i}, x_{4}^{(i)}\right)$ is in $F_{H}$, then $\left(x_{3}^{(i)}, x_{4}^{(i)}\right)$ is in $F_{H}$, and thus $\left(u_{i}, x_{4}^{(i)}\right)$ is not in $C_{\text {dir }}^{(H)}$. Since each vertex of $V(D)$ has in- and out-degree 1 in $F_{H}$ we have the following: 
Claim 2.4. No vertex of $V(D)$ is in $C_{d i r}^{(H)}$.

Claim 2.4 implies that

$$
V\left(C_{\text {dir }}^{(H)}\right) \subseteq V\left(Z_{1}\right) \cup \cdots \cup V\left(Z_{r}\right) \cup V\left(L_{1}^{(x)}\right) \cup \cdots \cup V\left(L_{r}^{(x)}\right) \cup V\left(L_{1}^{(y)}\right) \cup \cdots \cup V\left(L_{r}^{(y)}\right) .
$$

Claim 2.5. (I) $Z_{i} \subseteq V\left(C_{d i r}^{(H)}\right)$, for some $i \in\{1, \ldots, r\}$.

(II) If $x_{1}^{(i)} \in V\left(C_{d i r}^{(H)}\right)$, then $\left\{x_{1}^{(i)}, \ldots, x_{6}^{(i)}\right\} \subseteq V\left(C_{d i r}^{(H)}\right)$.

(III) If $y_{1}^{(i)} \in V\left(C_{d i r}^{(H)}\right)$, then $\left\{y_{1}^{(i)}, \ldots, y_{6}^{(i)}\right\} \subseteq V\left(C_{d i r}^{(H)}\right)$.

(IV) If $Z_{i} \subseteq V\left(C_{d i r}^{(H)}\right)$, then $Z_{i+1} \subseteq V\left(C_{d i r}^{(H)}\right)$, where indices are taken modulo $r$.

Proof. Since

$$
L_{1}^{(x)} \cup \cdots \cup L_{r}^{(x)} \cup L_{1}^{(y)} \cup \cdots \cup L_{r}^{(y)}
$$

is a collection of disjoint (non-directed) 6-cycles, (I) is true. The statements (II) and (III) are straightforward to verify e.g. from Figure 3, and statement (IV) follows easily from (II) and (III).

Since $C_{\mathrm{dir}}^{(H)}$ is a directed cycle of $H$ it follows from Claim 2.5 that for each $i=1, \ldots, r, C_{\mathrm{dir}}^{(H)}$ contains all vertices of $L_{i}^{(x)}$ or $L_{i}^{(y)}$. Moreover, $C_{\text {dir }}^{(H)}$ contains all the vertices $Z_{1} \cup \cdots \cup Z_{r}$, and no vertices of $V(D)$.

Now consider the graph $\hat{H}=H-V\left(C_{\text {dir }}^{(H)}\right)$. Clearly, $\hat{F}_{H}=F_{H}-V\left(C_{\text {dir }}^{(H)}\right)$ is a 2-factor of $\hat{H}$ such that each vertex of $V(D)$ has in and out-degree 1 in $\hat{F}_{H}$.

Consider an arc $(a, b)$ of $D$ that is not in any 2-cycle and the corresponding associated 4-cycle $Q_{a b}$ in $H$, and label the vertices of $Q_{a b}$ according to Figure 2. We need the following claim, which easily follows from from the fact that the degree of $c_{3}$ and $c_{4}$ is 2 .

Claim 2.6. It holds that $\left(a, c_{2}\right)$ is in $\hat{F}_{H}$ if and only if $\left(c_{1}, b\right)$ is in $\hat{F}_{H}$.

Now consider an arc $\left(u_{i}, v_{i}\right)$ that is in a 2-cycle of $D$. We shall also need the following claim which follows easily from the facts that $\hat{F}_{H}$ is a 2 -factor of $\hat{H}$ and that for each $i=1, \ldots, r, C_{\operatorname{dir}}^{(H)}$ contains all vertices of $L_{i}^{(x)}$ or $L_{i}^{(y)}$.

Claim 2.7. (I) If $\left(u_{i}, x_{4}^{(i)}\right) \in A\left(\hat{F}_{H}\right)$, then $\left(x_{5}^{(i)}, v_{i}\right) \in A\left(\hat{F}_{H}\right)$, and $\left\{\left(y_{3}^{(i)}, u_{i}\right),\left(v_{i}, y_{2}^{(i)}\right)\right\} \cap A\left(\hat{F}_{H}\right)=\emptyset$.

(II) If $\left(x_{5}^{(i)}, v_{i}\right) \in A\left(\hat{F}_{H}\right)$, then $\left(u_{i}, x_{4}^{(i)}\right) \in A\left(\hat{F}_{H}\right)$ and $\left\{\left(y_{3}^{(i)}, u_{i}\right),\left(v_{i}, y_{2}^{(i)}\right)\right\} \cap A\left(\hat{F}_{H}\right)=\emptyset$.

(III) If $\left(y_{3}^{(i)}, u_{i}\right) \in A\left(\hat{F}_{H}\right)$, then $\left(v_{i}, y_{2}^{(i)}\right) \in A\left(\hat{F}_{H}\right)$ and $\left\{\left(x_{5}^{(i)}, v_{i}\right),\left(u_{i}, x_{4}^{(i)}\right)\right\} \cap A\left(\hat{F}_{H}\right)=\emptyset$.

$(I V)$ If $\left(v_{i}, y_{2}^{(i)}\right) \in A\left(\hat{F}_{H}\right)$, then $\left(y_{3}^{(i)}, u_{i}\right) \in A\left(\hat{F}_{H}\right)$ and $\left\{\left(x_{5}^{(i)}, v_{i}\right),\left(u_{i}, x_{4}^{(i)}\right)\right\} \cap A\left(\hat{F}_{H}\right)=\emptyset$.

Now, using the two claims above and the fact that each vertex of $V(D)$ has in and out-degree 1 in $\hat{F}_{H}$, it is straightforward to verify that $\hat{F}_{H}$ yields a 2-cycle-free cycle factor in $D$ as in the last part of the proof of Theorem 1.8. This completes the proof of the theorem.

A proper edge coloring of a graph $G$ is a map $f: E(G) \rightarrow\{1,2,3, \ldots\}$ of positive integers to the edges of $G$ such that $f(e) \neq f\left(e^{\prime}\right)$ whenever $e$ and $e^{\prime}$ are adjacent edges. For the proof of Theorem 1.5 , we shall use the fact that the following problem is $\mathcal{N} \mathcal{P}$-complete [8]. An (edge) precoloring of a graph $G$ is a coloring of some of the edges of $G$. 


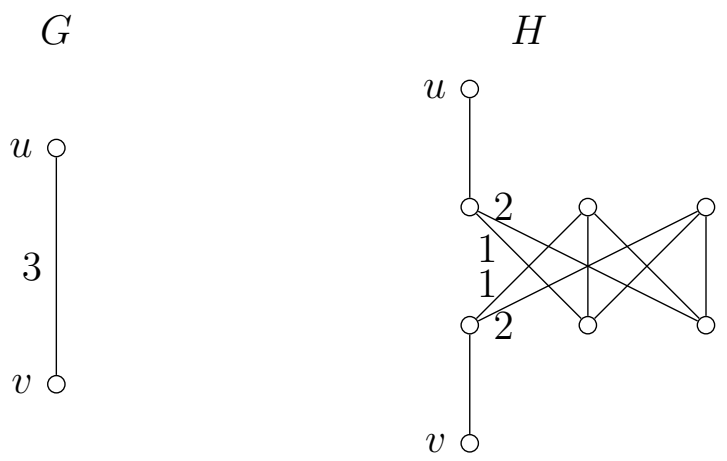

Figure 5: An edge precolored 3 in $G$ and the corresponding subgraph of $H$.

\section{Problem 2.2. Edge precoloring extension.}

Instance: A 3-regular bipartite graph $G$, a precoloring $f$ of $E^{\prime} \subseteq E(G)$.

Question: Can $f$ be extended to a proper edge coloring of $G$ using precisely 3 distinct colors?

Suppose now that $G$ is a cubic bipartite graph with a precoloring using 3 colors. By replacing every edge precolored 3 with the gadget in Figure 5, we obtain the graph $H$. It is easy to check that the precoloring of $G$ can be extended to a proper 3-edge coloring if and only if the precoloring of $H$ can be extended to a proper 3 -edge coloring. Hence, the following problem is also $\mathcal{N} \mathcal{P}$-complete.

\section{Problem 2.3. Edge precoloring extension with only two colors in the precoloring.}

Instance: A 3-regular bipartite graph $G$, a precoloring $f$ of $E^{\prime} \subseteq E(G)$ using only two distinct colors.

Question: Can $f$ be extended to a proper edge coloring of $G$ using precisely 3 distinct colors?

Proof of Theorem 1.5. We shall reduce Problem 2.3 above to the problem of determining if a 2-edge-colored bipartite graph has two disjoint perfect matchings $M_{1}$ and $M_{2}$ so that every edge of $M_{1}$ has color 1 .

Let $G$ be a 3-regular bipartite graph with some edges colored 1 and some edges colored 2. Denote this precoloring by $f$. We shall construct a bipartite graph $H$ with maximum degree 3 from $G$, where every edge in $H$ is colored 1 or 2 . Then we will argue that the precoloring $f$ can be extended to a proper 3-edge coloring of $G$ if and only if there are edge-disjoint perfect matchings $M_{1}$ and $M_{2}$ in $H$, such that $M_{1}$ only contains edges colored 1.

To this end, we define two 2-edge colored bipartite graphs $B$ and $C$ depicted in Figure 6, together with their compact notation. We say that $w$ and $z$, and $x$ and $y$ are endpoints of the graphs $B$ and $C$ respectively. 


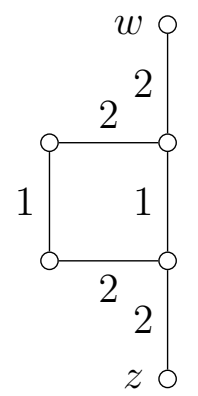

$C$

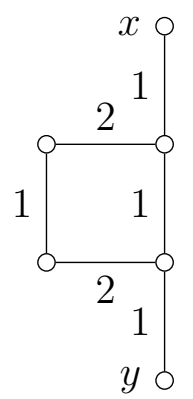

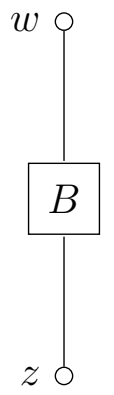

Compact notation for $C$

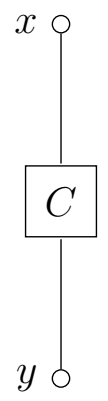

Figure 6: The graphs $B$ and $C$ and their compact notation.

Now we define the graph $H$ from $G$ by replacing all edges of $G$ according to the following procedure (see Figure 7). Let $e=u v$ be an edge of $G$. If

(a) $e$ is colored 1 , then $u v$ is replaced by a subgraph isomorphic to $C$ by identifying the vertices $x$ and $y$ of $C$ (see Figure 6) with the vertices $u$ and $v$, respectively;

(b) $e$ is colored 2, then the edge $u v$ is in $H$ and is colored 2 in $H$ as well;

(c) $e$ is uncolored and not adjacent to any colored edge of $G$, then $u v$ is in $H$ and we color it with 1;

(d) $e$ is uncolored and adjacent to some edge colored 2 but not adjacent to any edge colored 1 , then $u v$ is replaced by a subgraph isomorphic to $C$ by identifying the vertices $x$ and $y$ of $C$ (see Figure 6) with the vertices $u$ and $v$, respectively;

(e) $e$ is uncolored and adjacent to some edge colored 1 but not adjacent to any edge colored 2, then $u v$ is in $H$ and it is colored 2;

(f) $e$ is uncolored and adjacent to some edge colored 2 and also adjacent to some edge colored 1, then $u v$ is replaced by a subgraph isomorphic to $B$ by identifying $w$ and $z$ of $B$ (see Figure 6) with the vertices $u$ and $v$, respectively. 


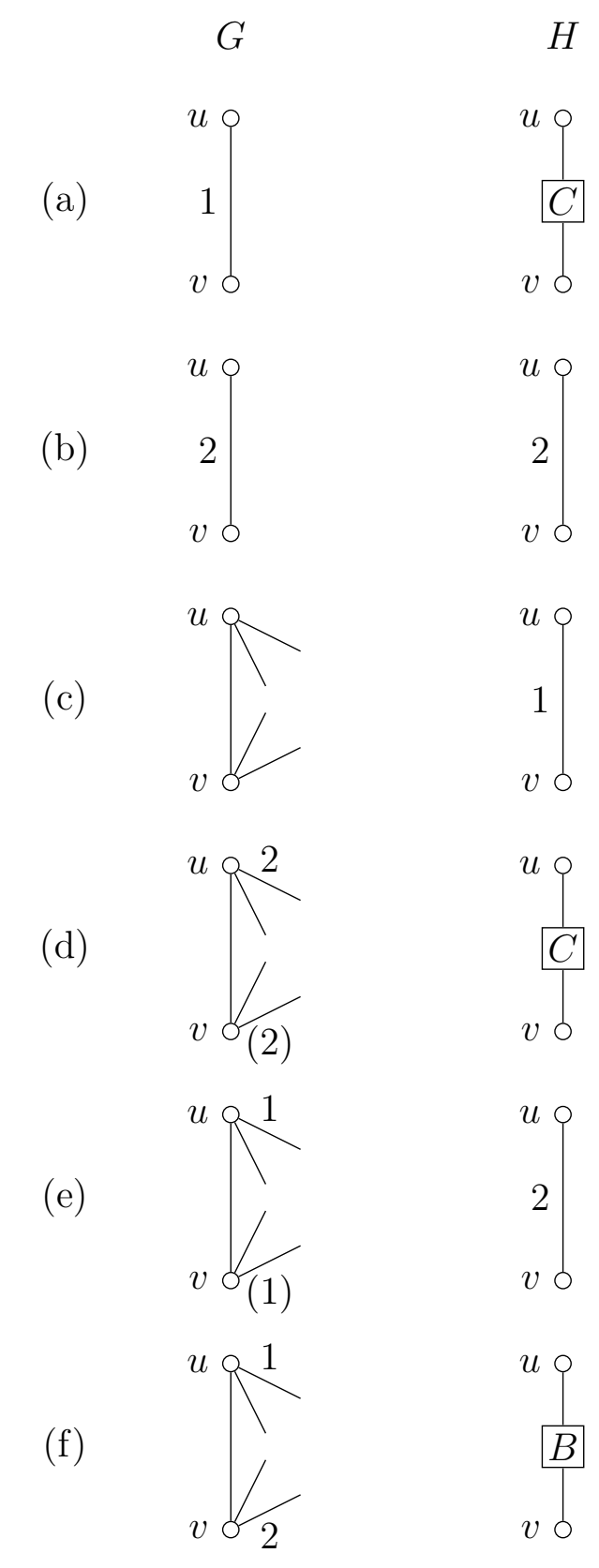

Figure 7: Constructing $H$ from $G$. 
The graph resulting from this process we denote by $H$ and we denote its edge coloring by $g$. We say that a subgraph isomorphic to $B(C)$ is a $B$-subgraph ( $C$-subgraph) of $H$ if it arises in $H$ by replacing an edge of $G$.

Suppose first that the precoloring $f$ of $G$ can be extended to a proper 3-edge coloring $f^{\prime}$ of $G$. We shall define the required matchings $M_{1}$ and $M_{2}$ in $H$.

We first define a set $\hat{M}_{1}$. Let $e_{1}=u_{1} v_{1}$ be an edge of $G$ with $f^{\prime}\left(u_{1} v_{1}\right)=1$. It follows from the construction of $H$, that either $u_{1} v_{1} \in E(H)$ and is colored 1, or $u_{1}$ and $v_{1}$ are endpoints of a $C$-subgraph $C_{1}$ in $H$. In the first case we include $u_{1} v_{1}$ in $\hat{M}_{1}$, and in the second case we include the edges of $C_{1}$ incident with $u_{1}$ and $v_{1}$ in $\hat{M}_{1}$; additionally, a third edge of $C_{1}$ is included in $\hat{M}_{1}$, so that these three edges form a perfect matching of $C_{1}$. By repeating this process for each $e$ in $G$ with $f^{\prime}(e)=1$, we obtain the matching $\hat{M}_{1}$ of $H$ that covers all vertices of $G$. It follows that the vertices of $H$ not covered by $\hat{M}_{1}$ lie on $B$ - or $C$-subgraphs of $H$. For each such subgraph with vertices uncovered by $\hat{M}_{1}$ we include two edges with color 1 in the set $M_{1}^{\prime}$, so that $\hat{M}_{1} \cup M_{1}^{\prime}$ is a perfect matching in $H$. We set $M_{1}=\hat{M}_{1} \cup M_{1}^{\prime}$.

Let us now construct $M_{2}$. Suppose that $e_{2}=u_{2} v_{2}$ is colored 2 under $f^{\prime}$. It follows from the construction of $H$, that $u_{2} v_{2} \in E(H)$ and is colored 1 or 2 under $g$. We include all edges $e \in E(G) \cap E(H)$ with $f^{\prime}(e)=2$ in $M_{2}$, and for each $C$ - and $B$-subgraph of $H$ we also include two edges with color 2 in $M_{2}$ so that $M_{2}$ is a perfect matching in $H$. Note that $M_{1} \cap M_{2}=\emptyset$, because $M_{1}$ only contains edges colored 1 (under $g$ ) from $B$ - and $C$-subgraphs and edges from $E(G)$ with color 1 under $f^{\prime}$. So $M_{1}$ and $M_{2}$ are the required perfect matchings of $H$.

Suppose now conversely that $H$ has disjoint perfect matchings $M_{1}$ and $M_{2}$ such that $M_{1}$ contains no edges colored 2 under $g$. We shall prove that there are disjoint perfect matchings $M_{1}^{\prime}$ and $M_{2}^{\prime}$ in $G$ such that $M_{1}^{\prime}$ contains all edges colored 1 under $f$, and $M_{2}^{\prime}$ contains all edges colored 2 under $f$, which yields the desired conclusion.

Consider a $B$-subgraph $B_{1}$ of $H$. Denote by $s$ and $t$, respectively, the vertices of $B_{1}$ of degree 2 that are not endpoints of $B_{1}$. Since $M_{1}$ is a perfect matching of $H$ that contains no edges colored 2 , the two edges colored 1 in $B_{1}$ are both in $M_{1}$. Furthermore, since $M_{2}$ is perfect, the two edges colored 2 that are incident with $s$ or $t$ are in $M_{2}$. Thus, we have the following:

Claim 2.8. If $u$ is an endpoint of a $B$-subgraph $B_{1}$ in $H$, then no edge of $\left(M_{1} \cup M_{2}\right) \cap E\left(B_{1}\right)$ is incident with $u$.

Now consider a $C$-subgraph $C_{1}$ of $H$. Denote by $a$ and $b$ the vertices of degree 2 in $C_{1}$ that are not endpoints of $C_{1}$; and by $a^{\prime}$ and $b^{\prime}$ the two vertices of degree three in $C_{1}$, where $a$ and $a^{\prime}$ are adjacent. Since $M_{1}$ is a perfect matching of $H$ that contains no edges colored 2, $a b \in M_{1}$. Moreover, since $M_{2}$ is perfect, the two edges in $C_{1}$ colored 2 are in $M_{2}$. Now consider the three edges of $C_{1}$ that are incident with $a^{\prime}$ or $b^{\prime}$. Since $M_{1}$ is perfect, it follows that either exactly two or one of these edges are in $M_{1}$. Thus, we have the following:

Claim 2.9. Let $u$ and $v$ be endpoints of one $C$-subgraph $C_{1}$ in $H$. Then no edge of $E\left(C_{1}\right) \cap M_{2}$ is incident with $u$ or $v$, and, moreover if $u$ is incident with an edge from $E\left(C_{1}\right) \cap M_{1}$ then $v$ is incident with an edge from $E\left(C_{1}\right) \cap M_{1}$.

We now construct $M_{2}^{\prime}$ from $M_{2}$ as follows: let $u_{2} \in V(G)$ and suppose that $e_{2}=u_{2} v_{2} \in M_{2}$. It follows from Claims 2.8 and 2.9 that $u_{2} v_{2}$ is not in any $B$ - or $C$-subgraph of $H$, so $u_{2} v_{2} \in E(G)$. Hence, by the construction of $H$, either $u_{2} v_{2}$ is uncolored under $f$, or has color 2 under $f$. Let $\hat{M}_{2}$ be the set of all edges $e$ of $M_{2}$ that are in $B$ - or $C$-subgraphs of $H$. We simply define $M_{2}^{\prime}$ by setting $M_{2}^{\prime}=M_{2} \backslash \hat{M}_{2}$. Let us verify that $M_{2}^{\prime}$ contains all edges precolored 2 in $G$. If $f(e)=2$, then $g(e)=2$, and any edge adjacent to $e$ in $G$ is replaced by a $B$ - or $C$-subgraph in $H$. So Claims 2.8 and 2.9 imply that $e \in M_{2}$, and thus $e \in M_{2}^{\prime}$. 
Now we construct $M_{1}^{\prime}$ from $M_{1}$ as follows: let $u_{1} \in V(G)$ and suppose that $e_{1}=u_{1} v_{1} \in M_{1}$. Let $\hat{M}_{1}$ be the set of all edges $e$ of $M_{1}$ that are in $B$ - or $C$-subgraphs, and which are not incident to endpoints of such subgraphs. It follows from Claim 2.8 that $e_{1}$ is not in any $B$-subgraph of $H$, and if $u_{1} v_{1}$ is in a $C$-subgraph, and thus $u_{1}$ is an endpoint of a $C$-subgraph $C_{1}$, then the other endpoint $w_{1}$ of $C_{1}$ is also incident with an edge from $M_{1} \cap E\left(C_{1}\right)$. For each such edge in $M_{1}$ we include the edge $u_{1} w_{1}$ in $M_{1}^{\prime}$. Note further that any edge of $M_{1} \backslash \hat{M}_{1}$ that is not in any $B$ - or $C$-subgraph of $H$ is in $G$. Thus, we include all such edges in $M_{1}^{\prime}$. Clearly, $M_{1}^{\prime}$ covers all vertices of $G$ and is disjoint from $M_{2}^{\prime}$. Let us verify that all edges precolored 1 in $G$ are in $M_{1}^{\prime}$. Let $e \in E(G)$ with $f(e)=1$. Then $e$ is replaced by a $C$-subgraph $C_{1}$ in $H$, and any edge that is adjacent to $e$ in $G$ is replaced by an edge colored 2 or a $B$-subgraph in $H$. Since $M_{1}$ only contains edges colored 1 under $g$ and contains no edge of a $B$-subgraph $B_{1}$ that is incident with an endpoint of $B_{1}$, it follows that the endpoints of $C_{1}$ are covered by edges from $M_{1} \cap E\left(C_{1}\right)$. Thus $e \in M_{1}^{\prime}$.

\section{Restricted decompositions of digraphs}

In this section we prove Theorem 1.9. The following is a well-known fact, see e.g. [1, Excercise 4.8].

Proposition 3.1. The arc set of a digraph $D=(V, A)$ can be decomposed into arc-disjoint directed cycles $C_{1}, \ldots, C_{p}$ for some $p$ if and only if we have $d_{D}^{+}(v)=d_{D}^{-}(v)$ for all vertices $v \in V$.

Proof of Theorem 1.9. Observe that if the arc set $A$ can be decomposed into arc-disjoint directed cycles, then the problem is trivial, so we can assume that is not the case. Consequently, if any vertex $v$ has $\left|d^{+}(v)-d^{-}(v)\right|>2$, there can be no solution as all directed cycles contribute the same to the in- and out-degree of any vertex. So by Proposition 3.1, $D$ is a yes-instance if and only if there is a cycle $C$ which covers all the vertices in $V^{+} \cup V^{-}$where $V^{+}=\left\{v \in V: d^{+}(v)=d^{-}(v)+2\right\}$ and $V^{-}=\left\{v \in V ; d^{-}(v)=d^{+}(v)+2\right\}$ and satisfying that every vertex $v \in V^{+}$has out-degree 2 in $C$, every vertex $v \in V^{-}$has in-degree 2 in $C$, and every vertex $v \in V(C) \backslash\left(V^{+} \cup V^{-}\right)$has in- and out-degree 1 in $C$. Note that after removing the arcs of such a cycle $C$ the resulting digraph $D^{\prime}$ will satisfy the condition in Proposition 3.1.

Now we show how to reduce the hamiltonian cycle problem for cubic bipartite graphs to our problem (this problem is well-known to be NP-complete [9])

Let $B=(X, Y ; E)$ be a cubic bipartite graph (note that $|X|=|Y|)$ and form the directed graph $D(B)$ as follows: Orient all edges of $E$ from $X$ to $Y$. Add one new vertex $s$ and the following arcs: all $\operatorname{arcs}$ from $s$ to $X$ as well as all arcs from $Y$ to $s$. The digraph $D(B)$ satisfies that $d^{+}(s)=d^{-}(s)$, all vertices $x \in X$ have $d^{+}(x)=d^{-}(x)+2$ and all vertices $y \in Y$ satisfy $d^{-}(y)=d^{+}(y)+2$. By the remarks above, if $D$ has the desired decomposition into arc-disjoint cycles $C_{1}, C_{2}, \ldots, C_{k}$ such that $C_{1}$ is the only non-directed cycle, then the arcs of $C_{1}$ must correspond to a hamiltonian cycle back in $B$. Conversely, if $C$ is a hamiltonian cycle of $B$, then by removing the corresponding arcs in $D(B)$ the resulting digraph $D^{\prime}$ has a decomposition into arc-disjoint directed 3-cycles all of which contain $s$ and one arc of a perfect matching from $X$ to $Y$.

\section{A polynomial instance of Problem 1.2}

An easy consequence of Hall's marriage theorem (see e.g. [1, Theorem 4.11.3]) is that every balanced bipartite graph on $n+n$ vertices with minimum degree at least $\frac{n}{2}$ has a perfect matching. It is worth noting that this implies that Problem 1.2 is solvable in polynomial time if the balanced bipartite graph $B$ on $n+n$ vertices has minimum degree at least $\frac{n}{2}+1$ : if this holds, then Problem 1.2 has 
a positive answer if and only if there is a matching $M_{1}$ containing only edges colored 1 , because if there is such a matching $M_{1}$, then $B-M_{1}$ has a perfect matching by applying Hall's theorem to $B-M_{1}$.

With a little more effort we can prove the following strengthening of this result:

Proposition 4.1. Problem 1.2 is solvable in polynomial time for balanced bipartite graphs on $n+n$ vertices with minimum degree at least $\frac{n}{2}$.

Proof of Proposition 4.1. Let $B$ be a bipartite graph with parts $V_{1}$ and $V_{2}$ both of which have size $n$. We may assume that $n \geq 6$, since smaller instances can surely be checked in polynomial time. We will deal with the cases that $n$ is even and $n$ is odd separately. By the above remark we may assume that $\delta(B)=\lceil n / 2\rceil$.

In the case when $n$ is odd we will prove that if Problem 1.2 has a negative answer for $B$, then either $B$ has no perfect matching with only edges of color 1 , or if $B$ has such a matching, then there are subsets $W_{1} \subseteq V_{1}$ and $W_{2} \subseteq V_{2}$ of size $\left\lceil\frac{n}{2}\right\rceil$ such that

(A.1) $B\left[W_{1} \cup W_{2}\right]$ is a one-factor,

(A.2) $B\left[W_{1} \cup V_{2} \backslash W_{2}\right]$ is complete bipartite,

(A.3) $B\left[W_{2} \cup V_{1} \backslash W_{1}\right]$ is complete bipartite, and

(A.4) for every perfect matching $M$ containing only edges of color 1 in $B$, every edge of $B\left[W_{1} \cup W_{2}\right]$ is contained in $M$.

In the case when $n$ is even we will prove that if Problem 1.2 has a negative answer for $B$, then either $B$ has no perfect matching $M_{1}$ with only edges of color 1 , or if $B$ has such a matching, then, by renaming $V_{1}$ and $V_{2}$ if necessary, there is a subset $W$ of $V_{1}$ of size $\frac{n}{2}+1$, and a maximal subset $X$ of $V_{2}$ of size $n / 2$ or $n / 2+1$ containing all vertices $x$ of $V_{2}$ with exactly one edge joining $x$ with a vertex of $W$. Moreover, $W$ and $X$ satisfy the following:

(B.1) the edge set of $B[W \cup X]$ is a matching of size $n / 2$ or $n / 2+1$,

(B.2) the subgraph of $B$ induced by $V_{1} \backslash W$ and $X$ is complete bipartite,

(B.3) if $X$ has size $n / 2+1$, then the subgraph of $B$ induced by $W$ and $V_{2} \backslash X$ is complete bipartite,

(B.4) if $X$ has size $n / 2$, then every perfect matching $M$ with only edges colored 1 contains each edge of $B[W \cup X]$,

(B.5) if $X$ has size $n / 2+1$, then every perfect matching $M$ with only edges colored 1 contains at least $n / 2$ edges from $B[W \cup X]$.

We first deal with the case when $n$ is odd.

Case 1: $n$ is odd.

If $B$ has no perfect matching with only edges of color 1 , then there is nothing to prove, so suppose that $B$ has such a matching $M_{1}$. Consider the graph $B^{\prime}=B-M_{1}$, and suppose that Problem 1.2 has a negative answer for $B$, in particular, there is no perfect matching in $B^{\prime}$. Then, by Hall's theorem there is a subset $W_{1}$ of $V_{1}$ such that $\left|N_{B^{\prime}}\left(W_{1}\right)\right|<\left|W_{1}\right|$. Since $\delta\left(B^{\prime}\right)=\lfloor n / 2\rfloor$, we have that $\left|W_{1}\right| \geq\lceil n / 2\rceil$. It is easy to see that if we choose a minimal set $W_{1}$ with this property, then $\left|W_{1}\right| \leq\lceil n / 2\rceil$. Hence, there is such a set $W_{1}$ of size exactly $\lceil n / 2\rceil$. We set $W_{2}=V_{2} \backslash N_{B^{\prime}}\left(W_{1}\right)$. 
Then $\left|W_{2}\right|=\lceil n / 2\rceil$, and it is easy to see that $\left|N_{B^{\prime}}\left(W_{2}\right)\right|=\lfloor n / 2\rfloor$. Since $\delta\left(B^{\prime}\right)=\lfloor n / 2\rfloor$, this implies that the subgraphs $B^{\prime}\left[W_{1} \cup N_{B^{\prime}}\left(W_{1}\right)\right]$ and $B^{\prime}\left[W_{2} \cup N_{B^{\prime}}\left(W_{2}\right)\right]$ of $B$ are complete bipartite; and thus each vertex of $W_{1}$ is matched to a vertex in $W_{2}$ under $M_{1}$, and thus $B\left[W_{1} \cup W_{2}\right]$ is a one-factor. Hence, we have proved that (A.1), (A.2) and (A.3) hold.

Suppose now that there is some matching $M_{1}^{\prime}$ in $B$ that does not contain every edge of $B\left[W_{1} \cup\right.$ $\left.W_{2}\right]$. Then $B^{\prime \prime}=B-M_{1}^{\prime}$ has some edge connecting a vertex $w_{1}$ of $W_{1}$ and a vertex $w_{2}$ of $W_{2}$. Since $B\left[W_{1} \cup\left(V_{2} \backslash W_{2}\right)\right]$ and $B\left[W_{2} \cup\left(V_{1} \backslash W_{1}\right)\right]$ are complete bipartite it follows that $B^{\prime \prime}-\left\{w_{1} w_{2}\right\}$ has a perfect matching, which contradicts that Problem 1.2 has a negative answer. We conclude that (A.4) holds.

Note that deciding whether $B$ has a perfect matching $M_{1}$ with only edges of color 1 can be done in polynomial time e.g. using flows (see [1, Section 4.11]). Assuming that $B$ has such a matching, the problem of finding either a perfect matching in $B-M_{1}$ or a set $W_{1}$ as in the argument above can be solved in polynomial time by well-known algorithms for constructing maximum matchings in bipartite graphs. Moreover, given the set $W_{1}$, the problem of verifying whether there is a set $W_{2}$, such that (A.1)-(A.4) hold, is clearly solvable in polynomial time.

\section{Case 2: $n$ is even.}

If $B$ has no matching with only edges of color 1 , then there is nothing to prove, so suppose that $B$ has such a matching $M_{1}$. Consider the graph $B^{\prime}=B-M_{1}$, and suppose that Problem 1.2 has a negative answer, that is, there is no perfect matching in $B^{\prime}$. Then, by Hall's theorem there is a set $A$ that is a subset of $V_{1}$ or $V_{2}$ such that $\left|N_{B^{\prime}}(A)\right|<|A|$. Since $\delta\left(B^{\prime}\right)=n / 2-1$, we have that $|A| \geq n / 2$. On the other hand, if we choose a minimal set $A$ with this property, then $|A| \leq n / 2$. Hence, there is such a set $A$ of size exactly $n / 2$ with $\left|N_{B^{\prime}}(A)\right|=n / 2-1$. Assume without loss of generality that $A \subseteq V_{2}$. Clearly $B^{\prime}\left[A \cup N_{B^{\prime}}(A)\right]$ is complete bipartite. We set $W=V_{1} \backslash N_{B^{\prime}}(A)$. Then $W$ has size $n / 2+1$ and $\left|N_{B^{\prime}}(W)\right|=n / 2$. Since $\delta\left(B^{\prime}\right)=n / 2-1$, it follows that all vertices in $A$ are matched to vertices in $W$ under $M_{1}$. Hence, the edge set of $B[A \cup W]$ is a matching.

Let $X \subseteq V_{2}$ be a maximal subset of vertices such that every vertex of $X$ has degree 1 in $B[X \cup W]$. Clearly $X$ contains $A$, and thus has size at least $n / 2$. On the other hand, since $B$ has minimum degree $n / 2,|X| \leq n / 2+1$.

Suppose first that $X$ has size $n / 2$, and thus $X=A$. Then (B.1) holds, and (B.2) as well, because $B\left[A \cup N_{B^{\prime}}(A)\right]$ is complete bipartite. Let us prove that every perfect matching $M_{1}$ with only edges colored 1 in $B$ contains all edges of $B[X \cup W]$, i.e. that (B.4) holds.

Suppose that there is a perfect matching $M_{1}^{\prime}$ in $B$ with only edges colored 1 such that $w x \notin M_{1}^{\prime}$, where $w \in W$ and $x \in X$. Set $B^{\prime \prime}=B-M_{1}^{\prime}$. It follows that the bipartite graph $B_{1}=B^{\prime \prime}[W \backslash$ $\left.\{w\} \cup V_{2} \backslash X\right]$ is balanced, and contains no isolated vertex. Moreover, every vertex of $W$ has degree at least $n / 2-2$ in $B_{1}$, and thus $B_{1}$ contains at least $n^{2} / 4-n$ edges. If $B_{1}$ has no perfect matching, then by Hall's theorem there is a subset $W^{\prime} \subseteq W$ of size $n / 2-1$, such that $\left|N_{B_{1}}\left(W^{\prime}\right)\right|=n / 2-2$. Consequently, there are two vertices $u_{1}, u_{2} \in V_{2} \backslash X$ of degree 1 in $B_{1}$. This implies that $B_{1}$ has at most $(n / 2-2)(n / 2-1)+2$ edges, a contradiction because $B_{1}$ has at least $n^{2} / 4-n$ edges and $n \geq 6$. Thus $B_{1}$ has a perfect matching $\hat{M}_{1}$.

Now consider the balanced bipartite graph $B_{2}=B^{\prime \prime}\left[X \backslash\{x\} \cup N_{B^{\prime}}(X)\right]$. Every vertex of $X$ has degree at least $n / 2-2$ in $B_{2}$. So provided that $n \geq 6, B_{2}$ has a perfect matching $\hat{M}_{2}$. This means that $\hat{M}_{1} \cup \hat{M}_{2} \cup\{x w\}$ is a perfect matching in $B^{\prime \prime}$, contradicting that Problem 1.2 has a negative answer. Thus we conclude that (B.4) holds.

Suppose now that $X$ has size $n / 2+1$. Recall that the edge set of $B[W \cup A]$ is a matching, and we have $A \subseteq X$. We claim that (B.1) holds. To wit, suppose that $x \in X \backslash A$ is adjacent to the same vertex in $W$ as a vertex $a \in A$; then, since $|W|=n / 2+1$, this means that there is some vertex $w \in W$ that has no neighbor in $X$, which means that $w$ has degree $n / 2-1$ in $B$, a contradiction. 
Hence, $B[W \cup X]$ is a one-factor.

That (B.2) and (B.3) hold follows easily from the facts that $\delta(B)=n / 2$ and $\left|V_{1} \backslash W\right|=\left|V_{2} \backslash X\right|=$ $n / 2-1$. Let us now prove that (B.5) holds.

Suppose that there is a perfect matching $M_{1}^{\prime}$ with only edges of color 1 such that $w_{1} x_{1} \notin M_{1}^{\prime}$ and $w_{2} x_{2} \notin M_{1}^{\prime}$, where $w_{i} \in W$ and $x_{i} \in X, i=1,2$. Set $B^{\prime \prime}=B-M_{1}^{\prime}$. It follows from (B.2) and (B.3) that $B_{3}=B^{\prime \prime}\left[W \backslash\left\{w_{1}, w_{2}\right\} \cup V_{2} \backslash X\right]$ and $B_{4}=B^{\prime \prime}\left[V_{1} \backslash W \cup X \backslash\left\{x_{1}, x_{2}\right\}\right]$ are balanced bipartite graphs with parts of size $n / 2-1$ where each vertex has degree at least $n / 2-2$. Hence, $B_{3}$ has a perfect matching $\hat{M}_{3}$ and $B_{4}$ has a perfect matching $\hat{M}_{4}$. Then $\hat{M}_{3} \cup \hat{M}_{4} \cup\left\{x_{1} w_{1}, x_{2} w_{2}\right\}$ is a perfect matching in $B^{\prime \prime}$, contradicting that Problem 1.2 has a negative answer. Hence, (B.5) holds.

As mentioned above, deciding whether $B$ has a perfect matching with only edges of color 1 can be done in polynomial time. Assuming that $B$ has such a matching, the problem of finding the set $W$ in the proof above can be solved in polynomial time by well-known algorithms for constructing maximum perfect matchings in bipartite graphs. The set $X$ can clearly also be found in polynomial time. Moreover, given the sets $W$ and $X$, the problem of verifying whether $W$ and $X$ satisfy that (B.1)-(B.5) hold is clearly solvable in polynomial time.

We conclude that Problem 1.2 is solvable in polynomial time for a balanced bipartite graph on $n+n$ vertices with minimum degree at least $n / 2$.

\section{References}

[1] J. Bang-Jensen and G. Gutin, Digraphs: Theory, Algorithms and Applications 2nd Ed., Springer Verlag, London 2009.

[2] J. Bang-Jensen and M. Kriesell, On the problem of finding disjoint cycles and dicycles in a digraph, Combinatorica 31(6) (2011) 639-668.

[3] J. Bang-Jensen, M.Kriesell, A. Maddaloni and S. Simonsen, Vertex-disjoint directed and undirected cycles in general digraphs, J. Combinatorial Theory, Ser B 106 (2014) 1-14.

[4] M. Bläser, B. Siebert, Computing Cycle covers without short cycles, Proc. 9th Ann. European Symp. on Algorithms (ESA), Lecture Notes in Comput. Sci. 2161, pages 369-379, 2001.

[5] J.A. Bondy and U.S.R. Murty, Graph Theory, Springer Graduate texts in Mathematics 244, Springer Verlag 2008.

[6] C. J. Casselgren, On avoiding some families of arrays, Discr. Math. 312 (2012), 963-972.

[7] Open problems collection from the program Graph, Hypergraphs and Computing at MittagLeffler Institute, http://www.mittag-leffler.se/sites/default/files/documents/problemcollection140911.pdf

[8] J. Fiala, NP-completeness of the edge precoloring extension problem on bipartite graphs, Journal of Graph Theory 43 (2003), pp. 156-160.

[9] M.R. Garey, D.S. Johnson and R.E. Tarjan, The planar Hamiltonian circuit problem is NPcomplete, Siam J. Comput. 5 (1976) 704-714.

[10] David Hartvigsen, Finding maximum square-free 2-matchings in bipartite graphs, Journal of Combinatorial Theory, Series B Volume 96, Issue 5, 2006, Pages 693705. 
[11] D. Hartvigsen, Extensions of Matching Theory, Doctoral thesis, Carnegie-Mellon University, 1984.

[12] L. Lovász, On graphs not containing independent circuits (in Hungarian), Mat. Lapok bf 16 (1965) 289-299.

[13] W. McCuaig, Intercyclic digraphs, In: Graph structure theory (Seattle WA, 1991), Contemp Math. 147, Amer. Math. Soc., Providence, RI (1993), 203-245. 